\title{
REPORTE DE CASO DE ENFERMEDAD DE POTT EN VÉRTEBRAS TORÁCICAS ALTAS EN UN NIÑO DE DOS AÑOS
}

\author{
Nathaly Cortez-Bazán,a, Jennifer R. Delgado²,a, Omar Galdos,a, Luis Huicho, 2,4,a
}

\begin{abstract}
RESUMEN
La enfermedad de Pott es un problema de salud en países en desarrollo y su diagnóstico en niños es un desafío. Presentamos el caso de un niño de dos años de edad, con enfermedad de Pott que compromete vértebras torácicas de T1 a T3. El cuadro clínico se caracterizó por dificultad para caminar, fiebre, tos y disnea. Al examen físico, se evidenció cifosis y prominencia ósea en la región cervicodorsal. Se obtuvo una prueba de tuberculina positiva y se aisló Mycobacterium tuberculosis en el cultivo del aspirado gástrico. La resonancia de columna vertebral mostró un absceso frio, destrucción de dos vértebras y compresión de la médula espinal. El paciente presentó mejoría con la terapia antituberculosa. Resaltamos la importancia de tener en cuenta la sospecha clínica para la detección temprana de esta condición, así como un inicio rápido del tratamiento antituberculoso, para evitar la discapacidad y mortalidad asociada a esta enfermedad.
\end{abstract}

Palabras clave: Tuberculosis vertebral; Diagnóstico; Tratamiento; Preescolar (fuente: DeCS BIREME).

\section{POTT'S DISEASE IN UPPER THORACIC VERTEBRAE IN A TWO-YEAR-OLD BOY: CASE REPORT}

\begin{abstract}
Pott's disease is a health problem in developing countries and its diagnosis in children is a challenge. Here we present the case of a two-year-old boy with Pott's disease involving T1 to T3 thoracic vertebrae. The clinical presentation was characterized by difficulty walking, fever, cough, and dyspnea. At physical examination, kyphosis and bony prominence were observed in the cervicodorsal area. A positive tuberculin test was obtained, and Mycobacterium tuberculosis was isolated via culture of the gastric aspiration sample. The spine MRI showed a chronic abscess, destruction of two vertebrae, and bone marrow compression. The patient experienced some improvement with anti-TB therapy. Here, we emphasize the importance of giving consideration to the clinical suspicion for the early detection of this condition, as well as a quick TB-treatment start so as to avoid the disability and mortality associated to this disease.
\end{abstract}

Keywords: Tuberculosis, spinal; Diagnosis; Treatment; Child, Preschool (source: MeSH NLM)

\section{INTRODUCCIÓN}

Laenfermedad de Pott (llamada también tuberculosis vertebral o espinal) es una forma de tuberculosis extrapulmonar que afecta a la columna vertebral. Las vértebras lumbares superiores y torácicas inferiores son las regiones más afectadas ${ }^{(1,2)}$. Los niños menores de cinco años, que hayan tenido contacto con personas con tuberculosis son afectados con mayor frecuencia ${ }^{(3)}$. La presentación clínica en niños es variable, siendo más frecuente la presencia de dolor en la zona afectada, asociado a cifosis y abscesos fríos. En estadios avanzados, puede llegar a comprimir la médula espinal. La progresión de la enfermedad de Pott es lenta e insidiosa. Síntomas constitucionales como fatiga, sudoración nocturna, mialgias, cambios de apetito y peso, solo están presentes en el $20 \%$ a $30 \%$ de los casos ${ }^{(2,4)}$. Las complicaciones de la

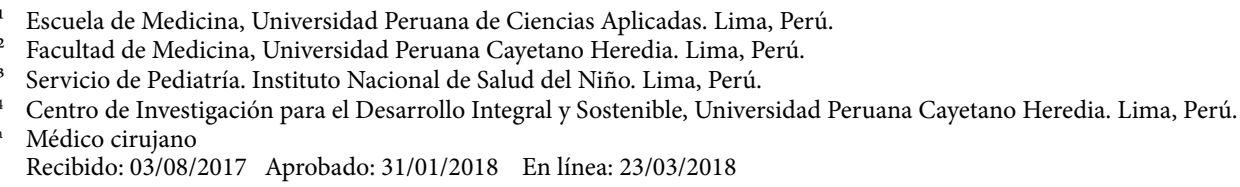


enfermedad son devastadoras e incluyen destrucción ósea, deformidad de la columna y disfunción neurológica severa ${ }^{(5)}$.

Según el reporte global de la Organización Mundial de la Salud (OMS) del 2017, se produjeron un millón de casos incidentes de tuberculosis en niños menores de 14 años, de los cuales $10 \%$ a $20 \%$ representan casos de tuberculosis extrapulmonar, de estos últimos, el $10 \%$ a $35 \%$ representan casos reportados de enfermedad de Pott ${ }^{(6)}$. Por otro lado, la proporción de casos de enfermedad de Pott en individuos infectados con VIH es comparable a la proporción de casos en individuos no infectados ${ }^{(7)}$. En el Perú, en el 2016, la incidencia de tuberculosis y de la enfermedad de Pott fue de 4700 casos y de 0,3 casos por cada cien mil niños menores de 14 años, respectivamente ${ }^{(6)}$. No obstante, es probable que la incidencia sea más alta, debido a la dificultad en el diagnóstico y a la escasa notificación de casos.

Si bien, la enfermedad de Pott puede considerarse poco frecuente, no se debe subestimar la urgencia de este problema, ya que está asociada a discapacidad severa y mortalidad alta si no es detectada y tratada a tiempo ${ }^{(8)}$. La evidencia sobre el manejo de estos casos en niños es limitada. Existe controversia sobre los regímenes terapéuticos e indicaciones quirúrgicas que deberían adoptarse.

Presentamos este caso de un niño de dos años con enfermedad de Pott, con la finalidad de promover en la comunidad médica la sospecha clínica de esta enfermedad, facilitando su identificación temprana, además de fomentar su notificación.

\section{REPORTE DE CASO}

Varón de 2,7 años previamente sano, proveniente de la ciudad de Lima. Recibió vacuna del Bacillus-CalmetteGuérin (BCG) al nacer. Tiene antecedentes de familiares con tuberculosis pulmonar activa (abuelos y un tío paterno), quienes fueron diagnosticados un mes antes del inicio del cuadro clínico del niño. Los contactos no vivían con el paciente, pero lo visitaban entre tres a cuatro veces por semana.

Cuatro semanas antes de acudir por emergencia y ser hospitalizado, el paciente presentó un cuadro caracterizado por fiebre intermitente, tos productiva, sibilantes y disnea moderada; luego de dos semanas, se le agrega debilidad de las extremidades inferiores de predominio distal. Además, presentó un incremento de su deterioro neurológico lo que ocasionó una marcada dificultad para la deambulación tres días antes de su ingreso al hospital.

Al examen físico, se evidenció la presencia de cifosis y una prominencia ósea en la región cervicodorsal. Además, se palparon múltiples linfadenopatías axilares y cervicales de aproximadamente $0,5 \mathrm{~cm}$ de diámetro. Al examen respiratorio se presentaron estertores en las bases de ambos campos pulmonares. Al examen neurológico, se observó paraparesia de miembros inferiores a predominio izquierdo, con incremento del tono muscular en la misma extremidad, también presentó disminución de los reflejos patelares y aquilianos del lado izquierdo con presencia de clonus. La sensibilidad bilateral se mantuvo conservada y el control de esfínteres preservado.

Los análisis de laboratorio mostraron un valor de proteína $\mathrm{C}$ reactiva de $2,71 \mathrm{mg} / \mathrm{dl}$ (normal $<10 \mathrm{mg} / \mathrm{dl}$ ) y una leucocitosis de $18780 / \mathrm{ml}$. La serología para VIH fue negativa. El test de tuberculina resultó positivo con $13 \mathrm{~mm}$ de induración.

La tomografía de tórax mostró consolidación del lóbulo inferior izquierdo y adenopatías múltiples de aspecto infeccioso (Figura 1), y la radiografía de tórax reveló opacidades en la misma localización. Ambos hallazgos sugirieron el diagnóstico de tuberculosis pulmonar. Además, se realizó cultivo bacteriológico automatizado BACTEC en aspirado gástrico, el cual resultó positivo para Mycobacterium tuberculosis.

Se inició terapia antituberculosa de acuerdo con los estándares específicos locales, incluyendo las cuatro drogas habituales (isoniacida, rifampicina, etambutol y pirazinamida). La tomografía de columna vertebral con contraste mostró abscesos osifluentes a nivel de T1 y T2 que se extendían hacia el canal medular y lo desplazaban posteriormente.

La resonancia magnética nuclear (RMN) de la columna vertebral reveló una colección inflamatoria tabicada entre los niveles vertebrales T1 y T3 en toda su extensión anterior y posterior, con compresión medular y lateralmente de ambas regiones paravertebrales (Figura 2); con destrucción de T2 y erosión del borde superior de T3 (Figura 3). Estos hallazgos fueron consistentes con el

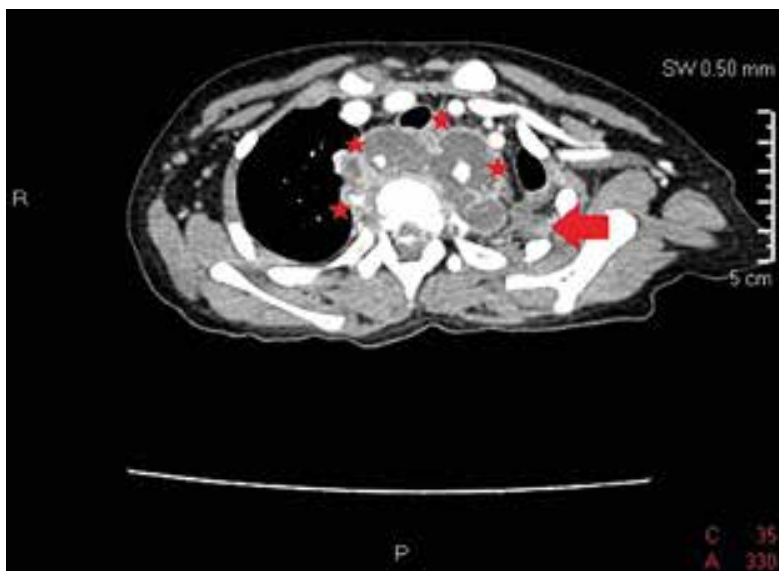

Figura 1. Corte axial de tomografía computarizada de tórax a nivel de T4. Se observa consolidación del lóbulo inferior izquierdo (flecha) y adenopatías mediastinales múltiples de aspecto infeccioso (estrellas). 


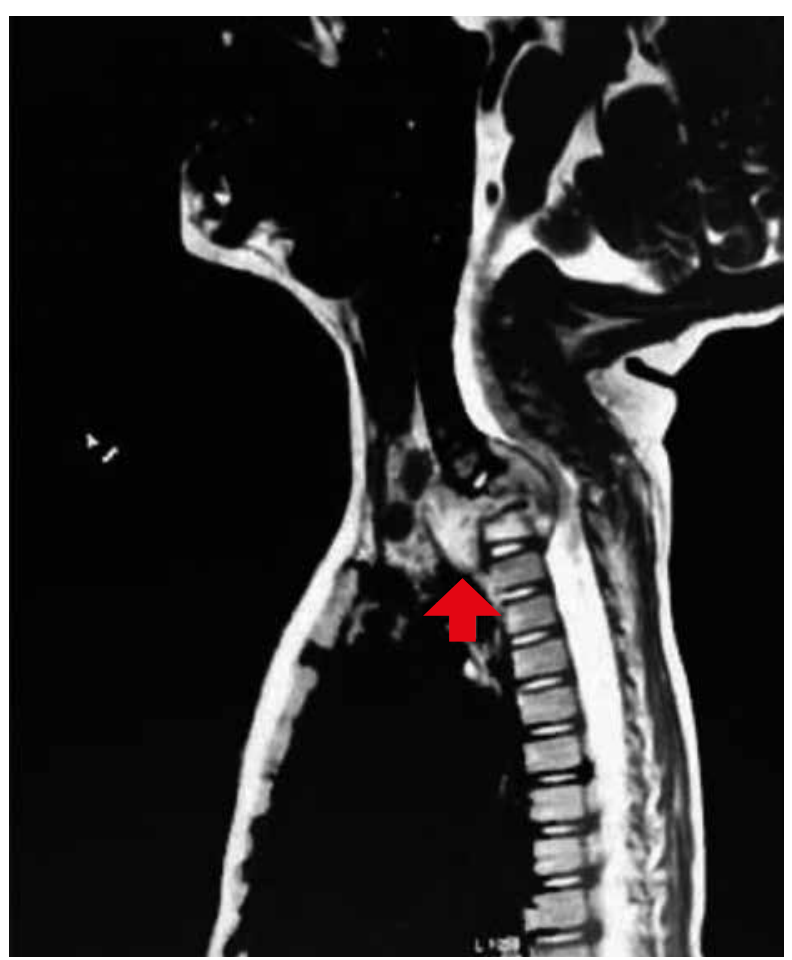

Figura 2. Corte sagital de resonancia magnética de columna vertebral superior. Se observa una colección inflamatoria entre T1 y T3. Además, se puede apreciar compresión medular.

diagnóstico de enfermedad de Pott. El paciente fue referido al departamento de neurocirugía para descompresión quirúrgica del canal medular. Mientras se encontraba en lista de espera para la cirugía, fue inmovilizado con un corsé tipo Minerva, fue dado de alta del servicio de Pediatría y continuó con terapia antituberculosa en forma ambulatoria.

A pesar de la severidad del cuadro clínico al ingreso, el paciente presentó mejoría clínica con la terapia antituberculosa. Luego de tres meses, la RMN de columna vertebral reveló resolución completa del absceso, el paciente logró retornar a una deambulación normal. Los contactos familiares con tuberculosis activa también recibieron tratamiento antituberculoso. Los padres y hermanos del

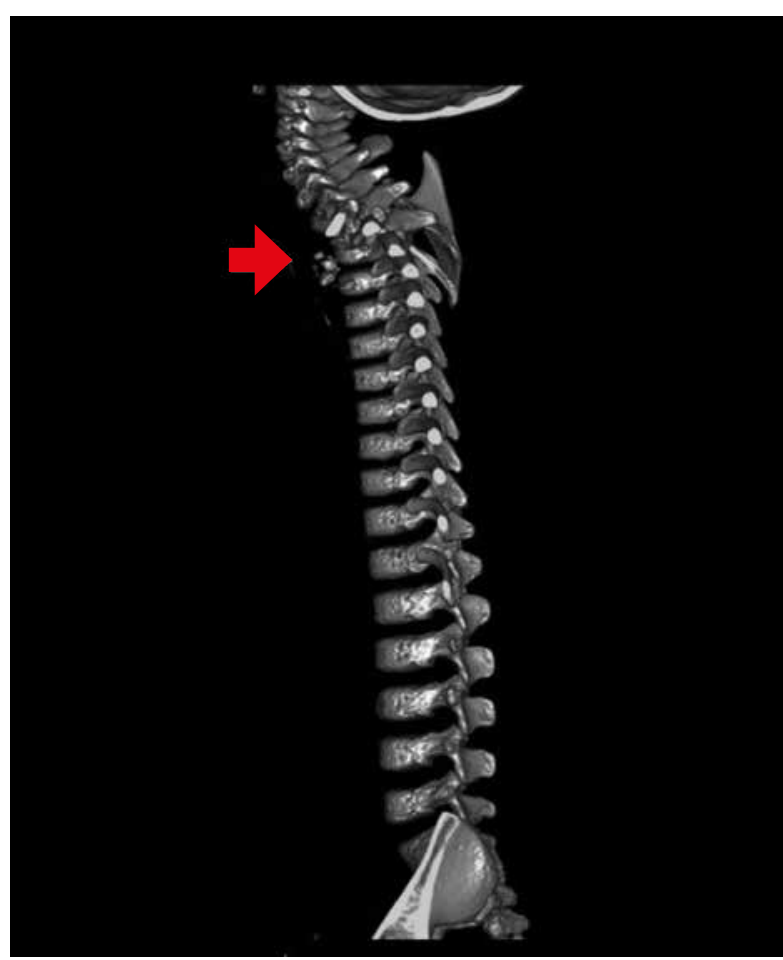

Figura 3. Reconstrucción digital en 3D de la resonancia magnética de la columna vertebral en su totalidad. Obsérvese la destrucción de las vértebras torácicas 1 y 3 .

paciente empezaron profilaxis antibiótica con isoniacida. En la tabla 1 se describe la evolución de la enfermedad.

\section{DISCUSIÓN}

La enfermedad de Pott en niños es una condición poco frecuente por ser de larga evolución, en la infección inicial ocurre una diseminación usualmente hematógena, que desarrolla una masa en tejido óseo con afectación espinal (4). En adultos, el $50 \%$ a $75 \%$ de los pacientes con tuberculosis osteoarticular y hasta el $33 \%$ a $50 \%$ de pacientes con tuberculosis espinal presentan un foco pulmonar primario activo $\mathrm{O}$ antecedente de tuberculosis pulmonar ${ }^{(9,10)}$. Sin embargo, la evidencia científica en población pediátrica es

Tabla 1. Evolución de la enfermedad de Pott en paciente varón de dos años

\begin{tabular}{lll}
\hline Día & Mes & Evolución de la enfermedad \\
\hline 16 & Junio 2016 & Contacto diagnosticado TB pulmonar activa \\
20 & Setiembre 2016 & Inicio de episodios febriles \\
01 & & Cifosis, dolor lumbar \\
10 & Octubre 2016 & Acude a emergencia del hospital por déficit motor en ambos miembros inferiores \\
13 & & Inicio de terapia antituberculosa \\
\hline 28 & Noviembre 2016 & Seguimiento y mejoría clínica postratamiento \\
\hline
\end{tabular}


escasa. Factores de riesgo asociados a esta complicación incluyen dificultad de acceso a servicios de salud, estados de inmunosupresión, y vivir en condición de hacinamiento ${ }^{(11)}$.

La columna vertebral es el lugar frecuentemente más afectado, representando a casi el $50 \%$ de los casos de tuberculosis ósea ${ }^{(12)}$. Un estudio retrospectivo en niños reveló que la columna vertebral torácica fue la más afectada ${ }^{(1)}$. Las características clínicas de la enfermedad de Pott son muy variadas e incluyen dolor local, espasmos musculares, abscesos fríos y deformidad espinal prominente. Los déficits neurológicos dependen del nivel comprometido. Si la médula torácica o lumbar es afectada, la función motora de los miembros superiores se mantiene, mientras que la debilidad de los miembros inferiores puede progresar a paraplejia, como en este caso ${ }^{(5)}$.

El diagnóstico de la enfermedad de Pott en la población infantil es complejo. El cultivo y análisis histopatológico del absceso frío son ideales, pero a veces son difíciles de realizar debido a barreras culturales, clínicas y socioeconómicas ${ }^{(13)}$. La RMN es el examen radiológico de elección, por ser más sensible que la radiografía y más específica que la tomografía. Por este motivo, se considera positivo el diagnóstico cuando existe una historia clínica compatible, asociado a imágenes concordantes en la resonancia magnética y una respuesta positiva al tratamiento antituberculoso (1). Uno de los hallazgos altamente sugestivos de enfermedad de Pott en la RMN, que predominó en este paciente, es la formación de abscesos y colecciones con expansión de tejido de granulación hacia el cuerpo vertebral ${ }^{(12)}$. En este caso, se confirmó el diagnóstico de tuberculosis pulmonar activa con el cultivo positivo en el aspirado gástrico, asociado a las características clínico radiológicas del paciente.

No existen guías estandarizadas para el manejo de enfermedad de Pott; sin embargo, varios estudios han demostrado que el $82 \%$ a $95 \%$ de los pacientes responden de manera favorable a la terapia con fármacos antituberculosos, por lo que este sigue siendo el tratamiento de elección en la población pediátrica ${ }^{(9,14)}$. Casi todas las drogas antituberculosas penetran bien en las lesiones tuberculosas vertebrales. Esta respuesta al tratamiento incluye disminución del dolor, mejoría en el déficit neurológico y corrección de la deformidad espinal ${ }^{(4)}$.

Debido a los riesgos serios de discapacidad y mortalidad y por las dificultades en la evaluación del tratamiento, la OMS recomienda nueve meses de tratamiento para la enfermedad de Pott ${ }^{(8)}$. Algunos autores recomiendan, además, la realización de intervención quirúrgica cuando ocurre déficit neurológico asociado a compresión medular. Sin embargo, una revisión de Cochrane sobre el uso de la intervención quirúrgica en adición al tratamiento antituberculoso, concluyó que no hay evidencia suficiente para recomendar la cirugía de rutina; ya que no se hallaron diferencias en la reducción del déficit neurológico, pérdida ósea, muerte por cualquier causa o nivel de actividad recuperado ${ }^{(15)}$. Cabe resaltar, que en Perú podrían existir otras variables como el acceso a servicios de neurocirugía, el tiempo prolongado de hospitalización y el riesgo de adquirir otras infecciones intrahospitalarias, que podrían limitar la realización y el éxito de la cirugía.

Por otro lado, la tuberculosis infantil siempre ha sido de difícil diagnóstico y en menores de dos años hay mayor riesgo de desarrollar complicaciones. Según el Centro Nacional de Epidemiología, Prevención y Control de Enfermedades, en el 2014 , el $63 \%$ de casos en menores de cinco años, estuvieron en contacto con pacientes con tuberculosis ${ }^{(3)}$. Por ello, es importante la investigación epidemiológica y el seguimiento de los contactos cuando se sospecha tuberculosis infantil. Para fortalecer las estrategias de diagnóstico y tratamiento oportuno, se necesita información acerca del comportamiento y las características de la enfermedad en este grupo etario, el cual se desarrolló en este reporte de caso.

En conclusión, consideramos que es importante tener en cuenta la sospecha clínica sobre esta condición en cualquier niño con contacto positivo para tuberculosis, no sólo en inmunosuprimidos. De esta manera, se podrá realizar un diagnóstico temprano y un pronto inicio de la terapia antibiótica antituberculosa combinada. Se requiere más evidencia para evaluar la prevalencia de las diferentes manifestaciones clínicas de esta condición en niños, y se necesitan guías de estandarización terapéutica para su manejo.

Consideraciones Éticas: Para la publicación del caso, se obtuvo el consentimiento informado de los padres del menor, además se ha eliminado la información identificativa relacionado al paciente.

Contribuciones de autoría: NCB, JRD, OG participaron en la recolección de datos. NCB y JRD participaron en el diseño del artículo y obtención de resultados. NCB, JRD y OG contribuyeron con el material de estudio, el análisis e interpretación de datos y la redacción del reporte. NCB, JRD, OG y LH participaron en revisión crítica y aprobación de la versión final.

Fuentes de financiamiento: autofinanciado.

Declaración de conflictos de interés: los autores declaran no tener conflictos de interés.

\section{REFERENCIAS BIBLIOGRÁFICAS}

1. Eisen S, Honywood L, Shingadia D, Novelli V. Spinal tuberculosis in children. Arch Dis Child. 2012;97(8):724-9. doi: 10.1136/archdischild-2011-301571.
2. Fuentes Ferrer M, Gutiérrez Torres L, Ayala Ramírez O, Rumayor Zarzuelo M, del Prado González N. Tuberculosis of the spine. A systematic review of case series.
Int Orthop. 2012;36(2):221-31. doi: 10.1007/s00264-011-1414-4.

3. Dirección General de Epidemiologia. Análisis de la situación epidemiológi- 
ca de la tuberculosis en el Perú 2015 [Internet]. Lima: DGE; 2016 [citado 20 de junio de 2017]. Disponible en: http://www.dge.gob.pe/portal/index. php?option $=$ com_content $\&$ view $=$ article\&id=599\&Itemid $=204$

4. Garg RK, Somvanshi DS. Spinal tuberculosis: A review. J Spinal Cord Med. 2011;34(5):440-54. doi: 10.1179/2045772311Y.0000000023.

5. Kotil K, Alan MS, Bilge T. Medical management of Pott disease in the thoracic and lumbar spine: a prospective clinical study.J Neurosurg Spine. 2007;6(3):222-8.

6. World Health Organization. Global tuberculosis report 2017 [Internet]. Geneva: WHO; 2017 [citado 13 de enero de 2018]. Disponible en: http://www.who. int/tb/publications/global_report/en/

7. Trecarichi EM, Di Meco E, Mazzotta V, Fantoni M. Tuberculous spondylodiscitis: epidemiology, clinical features, treatment, and outcome. Eur Rev Med Pharmacol Sci. 2012;16 Suppl 2:58-72.
8. World Health Organization. Guidelines for treatment of tuberculosis[Internet].Geneva: WHO. [citado 21 de junio de 2017]. Disponible en: http://www.who.int/tb/ publications/2010/9789241547833/en/

9. Arenas-Ruiz C, Díaz-Díaz A, Mesa-Monsalve JG, Trujillo M. Mal de Pott en un paciente pediátrico. Rev CES Med. 2014;28(2):253-62.

10. Schirmer P, Renault CA, Holodniy M. Is spinal tuberculosis contagious? Int J Infect Dis. 2010;14(8):e659-66. doi: 10.1016/j. ijid.2009.11.009.

11. Papan C, von Both U, Kappler M, Kammer B, Griese M, Huebner J. Pott's disease: a major issue for an unaccompanied refugee minor. Thorax. 2017;72(3):282-83. doi: 10.1136/thoraxjnl-2016-209468.

12. Rauf F, Chaudhry UR, Atif M, ur Rahaman M. Spinal tuberculosis: Our experience and a review of imaging methods. Neuroradiol J. 2015;28(5):498-503. doi: $10.1177 / 1971400915609874$.
13. Diagnostic Standards and Classification of Tuberculosis in Adults and Children. This official statement of the American Thoracic Society and the Centers for Disease Control and Prevention was adopted by the ATS Board of Directors, July 1999. This statement was endorsed by the Council of the Infectious Disease Society of America, September 1999. Am J Respir Crit Care Med. 2000;161(4 Pt 1):1376-95. doi: 10.1164/ajrccm.161.4.16141.

14. Agarwal A, Kant KS, Kumar A, Shaharyar A. One-year multidrug treatment for tuberculosis of the cervical spine in children.J Orthop Surg (Hong Kong). 2015;23(2):16873. doi: $10.1177 / 230949901502300210$.

15. Jutte PC, van Loenhout-Rooyackers JH. Routine surgery in addition to chemotherapy for treating spinal tuberculosis. Cochrane Database Syst Rev. 2006;(5):CD004532. doi: 10.1002/14651858.CD004532.pub2.

Correspondencia: Nathaly Cortez Bazán Teléfono: (+511) 3494043

Correo electrónico: nathalycortez@hotmail.com

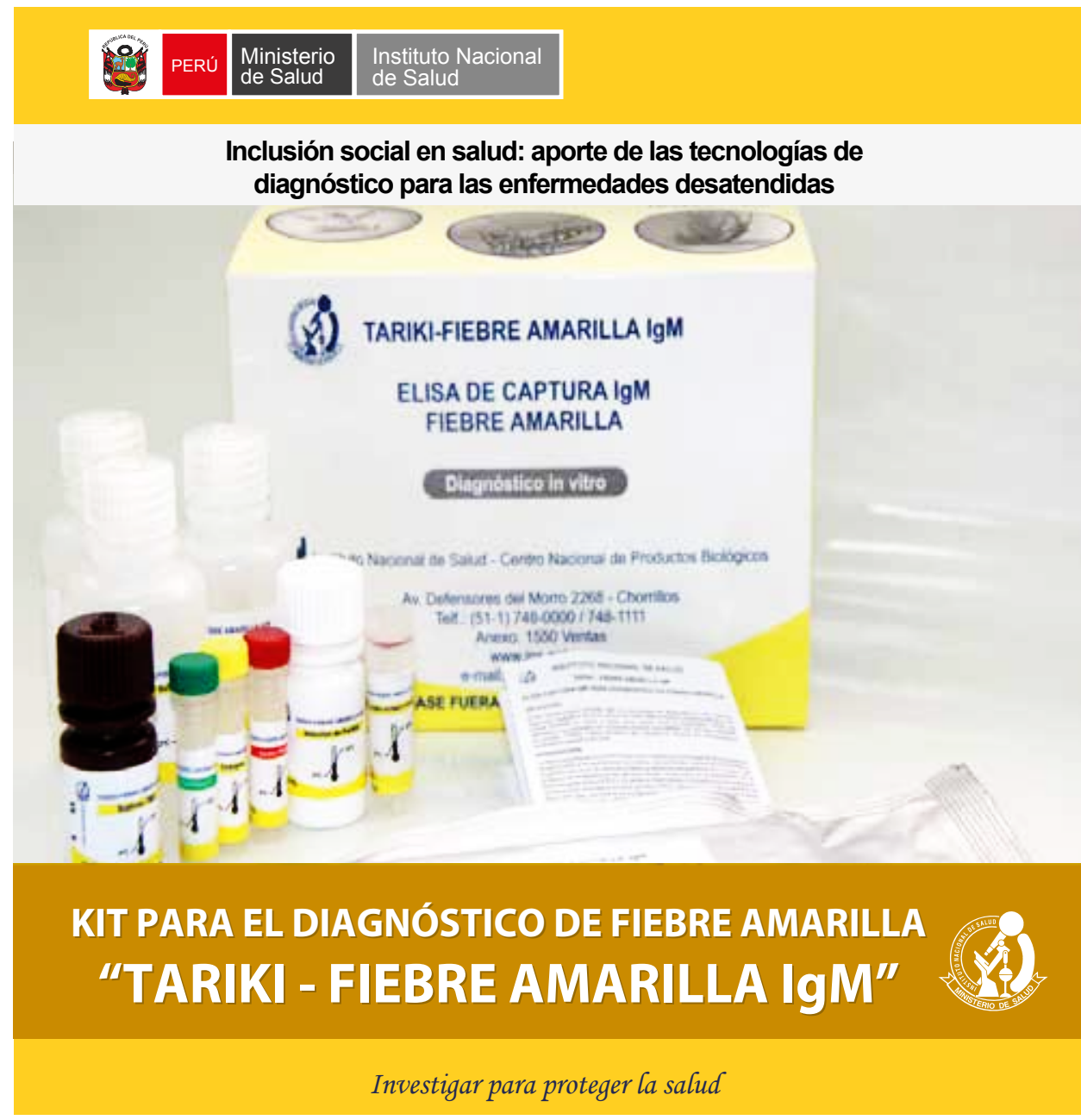

